\title{
Complaints and Inequality
}

by

Frank A. Cowell

STICERD, London School of Economics and Political Science

and

Udo Ebert

Universität Oldenburg

Discussion Paper

No. DARP 61

March 2002
Distributional Analysis Research Programme

The Toyota Centre Suntory and Toyota International Centres for Economics and Related Disciplines London School of Economics and Political Science Houghton Street London WC2A 2AE 


\section{Distributional Analysis Research Programme}

The Distributional Analysis Research Programme was established in 1993 with funding from the Economic and Social Research Council. It is located within the Suntory and Toyota International Centres for Economics and Related Disciplines (STICERD) at the London School of Economics and Political Science. The programme is directed by Frank Cowell. The Discussion Paper series is available free of charge and most papers are downloadable from the website. To subscribe to the DARP paper series, or for further information on the work of the Programme, please contact our Research Secretary, Sue Coles on:
Telephone: $\quad$ UK+20 79556678
Fax:
$\mathrm{UK}+2079556951$
Email:
s.coles@1se.ac.uk
Web site:
http://sticerd.lse.ac.uk/research/darp.asp 
Temkin $(1986,1993)$ sets out a philosophical basis for the analysis of income inequality that provides an important alternative to the mainstream welfarist approach. We show that the Temkin principles can be characterised by a parsimonious axiomatic structure and we use this structure to derive a new class of inequality indices and an inequality ordering. This class of indices has a family relationship to well-known measures of inequality, deprivation and poverty. The ordering is shown to have properties analogous to second-order dominance results.
\end{abstract}

Keywords: Inequality, complaints, transfer principle.

JEL classification: D63.

\title{
Acknowledgements:
}

We would like to acknowledge financial support by the Training and Mobility of Researcher Programme of the European Communties, grant \#ERBFMRXCT980248 (Living Standards, Inequality and Taxation), and to thank STICERD for hosting Ebert in order to facilitate our collaboration. Ralph Bayer helped greatly in preparation of the figures. We are grateful for comments from Yoram Amiel and Kurt Devooght.

(C) Frank A. Cowell and Udo Ebert. All rights reserved. Short sections of text, not to exceed two paragraphs, may be quoted without explicit permission provided that full credit, including $@$ notice, is given to the source.

Correspondence to: Professor F.A. Cowell, STICERD, LSE, Houghton Street, London WC2A 2AE. (f.cowell@lse.ac.uk) and to: Professor Udo Ebert, University of Oldenburg, D26111 Oldenburg, Germany, Email: (ebert@uni-oldenburg.de) 


\section{Introduction}

The mainstream literature on income inequality has used insights from related literatures - such as social welfare, information theory, risk analysis in order to motivate the analysis and to construct inequality measures. ${ }^{1}$ In some respects one can see inequality measurement approached in this way as a by-product of fundamental analysis developed for other purposes. By contrast the philosopher Larry Temkin has provided a basis for thinking about income inequality directly. His contributions (Temkin 1986, 1993) suggest that we may think of inequality simply as an aggregate of individual complaints experienced by individuals located in disadvantaged positions in the income distribution.

In this paper we move beyond the broad-brush suggestions of Temkin about the nature of these complaints and the way in which they may be aggregated in order to provide a rigorous analysis of a type of inequality index and inequality ordering that his approach entails. This is done by building a simple formal model of the income distribution and introducing a number of axioms that, although familiar from the inequality literature, encompass the distinctive Temkin approach. Sections 2 and 3 provide an overview of the issues and a description of our own methodology. Sections 4 and 5 establish the principal results on inequality and their implications. Section 6 introduces the associated ordering principle and demonstrates its relationship to the inequality indices. Section 7 examines some theoretical and practical extensions to the basic analysis.

\section{Background}

Constructing an approach to income inequality upon the notion of complaints about income distribution suggests that there may be connections with a number of existing intellectual strands in the income-distribution literature, including inequality, poverty and deprivation. This is the case and these will be discussed in sections 2.2 to 2.4 and elsewhere. But first we set out a summary of the novel contributions from Temkin himself.

\footnotetext{
${ }^{1}$ See Cowell (2000) for an overview.
} 


\subsection{Temkin: the essentials}

To oversimplify, the Temkin approach can be broken down into two components, as follows. ${ }^{2}$

Individual complaints. The fundamental concept required for the Temkin approach is that of an individual's complaint about income distribution. Typically this involves a person's welfare and that of a reference person or group (Temkin 1986, p. 104) although one could obviously express the complaint in terms of the person's income in comparison to that of others. It is an individualistic approach to the assessment of income distribution but one that is based on a primitive concept of differences rather than levels of utility or income. This is in clear contrast to the tradition of welfarism - see section 2.3 below.

The idea of a complaint raises two related questions: Who has a complaint? What is the reference group or point of reference? Temkin considers a number of possibilities but focuses attention principally on the following:

- Reference to the best-off person (BOP). Everyone but the person with the highest income has a legitimate complaint and everyone has the same reference point.

- Reference to all those who are better off (ATBO). Everyone but the best off has a legitimate complaint but persons at different positions in the income distribution make reference to different groups.

- Reference to average income (AVE).

Temkin discusses some specific well-known inequality measures in relation to each concept of complaint. However, two key problems remain open: whether the complaint approach induces a distinct coherent structure of inequality measurement and whether there may be other new inequality indices specific to the Temkin approach. Here we deal with these issues principally in the context of BOP. We will show that the approach developed for BOP can be extended to ATBO and possibly other concepts of reference group.

\footnotetext{
${ }^{2}$ For further discussion of the Temkin approach see Devooght (2001).
} 
Aggregation. The second component is the aggregation of complaints over the population. Again, more than one method of aggregation is discussed by Temkin: essentially inequality is to be regarded as either the simple or weighted sum of these complaints. Where a weighted approach is used, the weights increase with the size of the complaint (Temkin 1986, p.113).

The aggregation has normative significance because Temkin wants to interpret the overall complaint in the community as some kind of social bad. However the issue remains open as to whether a precise system of aggregation is implied by a specific set of ethical principles; this issue is also addressed in our axiomatic treatment.

\subsection{Complaint, Deprivation, Envy}

The Temkin approach finds some echoes in the literature on the mainstream literature on the assessment of income distribution. For example the idea of "complaint" may suggest that this is the concept of envy under utilitarianism. However the two concepts are distinct in nature because the complaint is considered to exist independently of the way a disadvantaged person may feel about it.

"To say that the best-off have nothing to complain about is in no way to impugn their moral sensibilities. They may be just as concerned about the inequality in their world as anyone else. Nor is it to deny that, insofar as one is concerned about inequality, one might have a complaint about them being as well of as they are. It is only to recognize that, since they are at least as well off as every other member of their world, they have nothing to complain about. Similarly, to say that the worst-off have a complaint is not to claim that they will in fact complain (they may not). It is only to recognize that it is a bad thing (unjust or unfair) for them to be worse off than the other members of their world through no fault of their own" - (Temkin 1986, p.102).

Secondly the description of a complaint suggests that there may be a logical relation to relative deprivation (Runciman 1966). The ATBO approach to complaint is indeed closely related but we disagree with Chakravarty (1998)'s remark that "the interpretation of relative deprivation in terms of income differences is formally equivalent to the Temkin (1986) approach to inequality measurement." First, Temkin's approach goes beyond the conventional 
concept of individual relative deprivation to encompass other concepts of complaint, notably BOP. Second, we will see that arising from Temkin's approach there is a large class of inequality measures that has not been explored by the deprivation literature.

\subsection{Welfare}

The main body of literature on the assessment of income distribution in terms of inequality is typically based on a welfarist and individualistic approach to distributional issues - see for example the seminal paper of Atkinson (1970). However the framework of inequality analysis that emerges from this is typically unsuitable for interpretation within the language of complaints (Temkin 1993, pages 135-141). Nevertheless a welfare interpretation of the complaints approach is important: Temkin is explicit that he is concerned which distribution is "better" rather than which exhibits greater or less inequality. It seems appropriate to examine the welfare implications of the complaints approach and, where possible try to reconcile it with the conventional welfare-economic approach to income distribution.

\subsection{The transfer principle}

The final strand that is relevant to our discussion concerns the principle that has been at the heart of mainstream inequality analysis for several decades.

The transfer principle has its origin in the work of Pigou (1912). However, Pigou confined his discussion to a two-person case where the distributional implications are straightforward. Dalton (1920) generalised the approach in a manner that has become the foundation for most of the modern work on distributional analysis: any transfer from a person to someone who is richer must increase inequality. However it has been argued that the Dalton extension of the Pigou approach is overly strong. Experimental evidence suggests that people's views on distributional rankings may violate the Dalton principle although being consistent with the elementary Pigou approach (Amiel and Cowell 1998, 1999). In what follows we provide a step towards addressing this problem by introducing more than one type of "transfer principle." 


\section{The approach}

We assume that the problem is one of evaluating and comparing income distributions in a finite fixed-sized population with more than three members and that income can take any real value. Throughout the following we will work with vectors of ordered incomes.

\subsection{Notation}

Let $\Omega_{n}^{*}$ be the set of ordered $n$-vectors:

$$
\Omega_{n}^{*}:=\left\{\mathbf{x}: \mathbf{x} \in \mathbb{R}^{n}, x_{1} \leq x_{2} \leq \ldots \leq x_{n}\right\}
$$

and define:

$$
\Omega_{n}:=\left\{\mathbf{x}: \mathbf{x} \in \Omega_{n}^{*}, x_{n-1}<x_{n}\right\} .
$$

An income distribution is given by

$$
\mathbf{x}:=\left(x_{1}, x_{2}, \ldots, x_{n}\right) \in \mathbb{D}
$$

where $\mathbb{D} \subseteq \Omega_{n}^{*}$ and $n>3$. Define $r=r(\mathbf{x})$ where

$$
r(\mathbf{x}):=\min \left\{i: x_{i}=x_{n}\right\}
$$

This device allows us to handle in a natural way the case where there are multiple persons at the very top of the distribution. Write $\mathbf{1}$ for the $n$ vector $(1,1, \ldots, 1)$ and $\mathbf{x}_{-i}(\alpha)$ for the vector $\mathbf{x}$ modified by replacing the $i$ th component by $\alpha$ :

$$
\mathbf{x}_{-i}(\alpha):=\left(x_{1}, \ldots, x_{i-1}, \alpha, x_{i+1}, \ldots, x_{n}\right)
$$

such that $x_{i-1} \leq \alpha \leq x_{i+1}$. We will also use $\mathbf{x}_{i j}(\delta)$ to denote the vector $\mathbf{x}$ modified by increasing the $i$ th component by $\delta$ and decreasing the $j$ th component by $\delta$.

Definition 1 An inequality measure is a function $T: \mathbb{D} \rightarrow \mathbb{R}_{+}$.

Definition 2 For any $\mathbf{x} \in \mathbb{D}$ and any positive $i<r$, where $r>1$ is given by (3) $a$ transfer from the richest is a transformation $\mathbf{x} \mapsto \mathbf{x}_{i r}(\delta)$ where $\delta>0$ is such that $x_{i}+\delta \leq x_{i+1}$ and $x_{r-1} \leq x_{r}-\delta$. 
Definition 3 For any $\mathbf{x} \in \mathbb{D}$ and any pair $(i, j)$ satisfying $0<i<j<r a$ progressive transfer is a transformation $\mathbf{x} \mapsto \mathbf{x}_{i j}(\delta)$ where $x_{i}+\delta \leq x_{i+1}$ and $x_{j}-\delta \geq x_{j-1}$.

Note the explicit distinction between definitions 2 and 3 - transfers from the richest (where the cause of the "complaint" arises) to the rest of the community and richer-to-poorer transfers within the group of complainants.

\subsection{Axioms}

The following six axioms are based on those that are standard in the literature on inequality and related issues; ${ }^{3}$ they are used to endow the class of complaint-regarding inequality measures with an appropriate structure:

Axiom 1 Continuity. $T$ is continuous.

Axiom 2 Monotonicity. For $\mathbf{x} \in \mathbb{D}$ and $i<r(\mathbf{x}) \quad T$ is strictly decreasing in $x_{i}$.

Axiom 3 Independence. Let $\mathbf{x}, \mathbf{y} \in \mathbb{D}$ be such that $T(\mathbf{x})=T(\mathbf{y}), r(\mathbf{x})=$ $r(\mathbf{y})=r>2$ and $x_{r}=y_{r}$. Then, for any $i<r, x_{i}=y_{i} \Rightarrow$

$\forall \alpha \in\left[x_{i-1}, x_{i+1}\right] \cap\left[y_{i-1}, y_{i+1}\right]$ and $\mathbf{x}_{-i}(\alpha), \mathbf{y}_{-i}(\alpha) \in \mathbb{D}: T\left(\mathbf{x}_{-i}(\alpha)\right)=T\left(\mathbf{y}_{-i}(\alpha)\right)$.

Axiom 4 Linear homogeneity. $\forall \lambda>0$ :

$$
T(\lambda \mathbf{x})=\lambda T(\mathbf{x}) .
$$

Axiom 5 Translation invariance. $\forall \alpha \in \mathbb{R}$ :

$$
T(\mathbf{x})=T(\mathbf{x}+\alpha \mathbf{1}),
$$

where $\mathbf{1}:=(1,1, \ldots, 1)$.

\footnotetext{
${ }^{3}$ For example, in motivating an analysis of the standard FGT (Foster et al. 1984) poverty measures Ebert and Moyes (2002) use continuity, monotonicity, linear homogeneity and translation invariance; likewise, in an axiomatisation of individual deprivation, Ebert and Moyes (2000) use these axioms and independence. The concept of independence in Ebert and Moyes (2002) is somewhat different since they do not use the concept of income rank.
} 
Axiom 6 Normalisation. $T(0, \ldots, 0,1)=1$

Axiom 2 formalises the idea that - for everyone other than the richest - an individual's complaint is reduced if his income is increased. Axiom 3 implies the following about two worlds with identical inequality and in which person with income-rank $i$ has the same complaint: varying that person's income by a small amount will have an identical impact on inequality in the two worlds. Clearly Axioms 4 and 5 imply that if all incomes double then so does the aggregate complaint, but adding a dollar simultaneously to everyone's resources (whether perpetrator or victim) leaves complaints untouched. ${ }^{4}$ Axioms 1 and 6 are technical requirements to ensure that the resulting inequality measure is well-defined.

The final two axioms focus on the two main ethical issues concerning complaint-regarding inequality measures.

Axiom 7 Transfers from the richest. $T$ satisfies the principle of transfers from the richest if

$$
T\left(\mathbf{x}_{i r}(\delta)\right)<T(\mathbf{x})
$$

where $\mathbf{x}_{i r}(\delta) \in \mathbb{D}$ and $i, r, \delta$ satisfy the conditions in Definition 2.

Axiom 8 Progressive transfers. T satisfies the principle of progressive transfers if

$$
T\left(\mathbf{x}_{i j}(\delta)\right)<T(\mathbf{x})
$$

where $\mathbf{x}_{i j}(\delta) \in \mathbb{D}$ and $i, j, r, \delta$ satisfy the conditions in Definition 3.

\section{Inequality: main results}

The analysis of inequality and complaints interpreted as BOP requires careful consideration of the set of income distributions. In section 4.1 we concentrate first on the special case where there is literally a single "Best-off Person" $\mathbb{D}=\Omega_{n}$ in (2). This is of course rather limiting; so in section 4.2 we examine the way in which some of the results can be extended to the case where there may be many persons with the topmost income.

\footnotetext{
${ }^{4}$ See, for example, Temkin (1986), p. 106, (1993) p. 26. However, Temkin (1993), chapter 6 appears to contradict this and this point is discussed in section 7.1 below.
} 


\subsection{Pure BOP}

Here one has $r(\mathbf{x})=n$. We proceed by first characterising the general structure of complaint-based inequality measures according to the principles set out in section 3.2 and then examining the general structure in the light of the two concepts of the transfer principle.

Proposition 1 Assume that $\mathbb{D}=\Omega_{n}$; then $T$ satisfies Axioms 1 to 6 if and only if there are $w_{k}>0, k=1, \ldots, n-1, \sum_{k=1}^{n-1} w_{k}=1$ and $\varepsilon \in \mathbb{R}$ such that

$$
\begin{aligned}
T_{\varepsilon}(\mathbf{x}) & =\left[\sum_{k=1}^{n-1} w_{k}\left[x_{n}-x_{k}\right]^{\varepsilon}\right]^{\frac{1}{\varepsilon}} \text { for } \varepsilon \neq 0 \\
& =\prod_{k=1}^{n-1}\left[x_{n}-x_{k}\right]^{w_{k}} \text { for } \varepsilon=0
\end{aligned}
$$

for $\mathbf{x} \in \mathbb{D}$.

Proof. From Theorem 5.5 of Fishburn (1970) continuity, monotonicity, independence and $n>3$ imply that there are monotonic functions $f_{0}, f_{1}, \ldots$, $f_{n-1}: \mathbb{R}^{2} \rightarrow \mathbb{R}$ such that

$$
T(\mathbf{x})=f_{0}^{-1}\left(\sum_{k=1}^{n-1} f_{k}\left(x_{k}, x_{n}\right), x_{n}\right)
$$

where $f_{0}^{-1}$ is the inverse with respect to the first argument. Using translation invariance (5) and setting $\alpha=-x_{n}$ we get

$$
T(\mathbf{x})=T\left(x_{1}-x_{n}, \ldots, x_{n-1}-x_{n}, 0\right)
$$

or

$$
\begin{aligned}
T(\mathbf{x}) & =f_{0}^{-1}\left(\sum_{k=1}^{n-1} f_{k}\left(x_{k}-x_{n}, 0\right), 0\right) \\
& =g_{0}\left(\sum_{k=1}^{n-1} g_{k}\left(x_{k}-x_{n}\right)\right)
\end{aligned}
$$


where $g_{0}(x):=f_{0}^{-1}(x, 0)$ and $g_{k}(x):=f_{k}(x, 0)$ for $k=1, \ldots, n-1$. By monotonicity there exist strictly increasing $h_{0}, h_{1}, \ldots, h_{n-1}$ such that $h_{1}, \ldots$, $h_{n-1}$ are defined on $\mathbb{R}_{++}$and

$$
T(\mathbf{x})=h_{0}\left(\sum_{k=1}^{n-1} h_{k}\left(x_{n}-x_{k}\right)\right)
$$

Now impose linear homogeneity (4). It is equivalent to

$$
\frac{1}{\lambda} h_{0}\left(\sum_{k=1}^{n-1} h_{k}\left(\lambda\left[x_{n}-x_{k}\right]\right)\right)=h_{0}\left(\sum_{k=1}^{n-1} h_{k}\left(x_{n}-x_{k}\right)\right)
$$

Define

$$
\begin{gathered}
H_{0}^{\lambda}(x):=\frac{1}{\lambda} h_{0}(x) \\
H_{k}^{\lambda}(x):=h_{k}(\lambda x), \text { for } k=1, \ldots, n-1 .
\end{gathered}
$$

Then (10) becomes

$$
H_{0}^{\lambda}\left(\sum_{k=1}^{n-1} H_{k}^{\lambda}\left(x_{n}-x_{k}\right)\right)=h_{0}\left(\sum_{k=1}^{n-1} h_{k}\left(x_{n}-x_{k}\right)\right)
$$

Theorem 2, Aczél (1966), p. 290 implies that

$$
H_{k}^{\lambda}(x)=a_{k}(\lambda) h_{k}(x)+b_{k}(\lambda)
$$

for $k=1, \ldots, n-1$ and similarly for $H_{0}^{\lambda}(x)$.

Using Theorem 2.7.3 in Eichhorn (1978) yields that there are $\beta_{k} \neq 0$, $\varepsilon_{k} \neq 0$ and $\gamma_{k} \in \mathbb{R}$ such that

$$
\left.\begin{array}{l}
h_{k}(x)=\beta_{k} \ln x+\gamma_{k} \\
a_{k}(\lambda)=1 \\
b_{k}(\lambda)=\gamma_{k} \ln \lambda
\end{array}\right\} \text {. }
$$

or

$$
\left.\begin{array}{l}
h_{k}(x)=\beta_{k} x^{\varepsilon_{k}}+\gamma_{k} \\
a_{k}(\lambda)=\lambda^{\varepsilon_{k}} \\
b_{k}(\lambda)=\gamma_{k}\left[1-\lambda^{\varepsilon_{k}}\right]
\end{array}\right\} .
$$


Thus there exist $\beta_{0}$ and $w_{1}, \ldots, w_{n-1}$ such that

$$
T(\mathbf{x})=\left\{\begin{array}{cc}
\beta_{0}\left[\sum_{k=1}^{n-1} w_{k}\left[x_{n}-x_{k}\right]^{\varepsilon}\right]^{\frac{1}{\varepsilon}} & \text { for } \varepsilon \neq 0 \\
\beta_{0} \prod_{k=1}^{n-1}\left[x_{n}-x_{k}\right]^{w_{k}} & \text { for } \varepsilon=0
\end{array}\right.
$$

where $\beta_{0}>0, \sum_{k=1}^{n-1} w_{k}=1, w_{k}>0$. (We obtain $\varepsilon_{1}=\varepsilon_{2}=\ldots=\varepsilon_{n-1}=\varepsilon$ and $\varepsilon_{0}=1 / \varepsilon$ because of linear homogeneity. $\sum w_{k}=1$ can be derived by choosing $\beta_{0}$ appropriately.)

Normalization implies that $\beta_{0}=1$.

It is immediate from $(8,9)$ that each member of the class $T_{\varepsilon}$ is differentiable. Furthermore we have the following result:

Proposition 2 Assume that $\mathbb{D}=\Omega_{n}$; then, for all $\varepsilon, T_{\varepsilon}$ satisfies the principle of transfers from the richest.

Proof. Consider $T_{\varepsilon}\left(\mathbf{x}_{i n}(\delta)\right)$ for some $i$ such that $x_{i}<x_{n}$. Given the differentiability of $(8,9)$ the effect of a transfer from the richest can be found from:

$$
\left.\frac{\partial T_{\varepsilon}\left(\mathbf{x}_{i n}(\delta)\right)}{\partial \delta}\right|_{\delta=0}=-T_{\varepsilon}(\mathbf{x})^{1-\varepsilon}\left[\sum_{k=1}^{n-1} w_{k}\left[x_{n}-x_{k}\right]^{\varepsilon-1}+w_{i}\left[x_{n}-x_{i}\right]^{\varepsilon-1}\right]
$$

Clearly this is negative, as required by Axiom 7.

However satisfaction of the principle of progressive transfers requires a further restriction on the class of indices:

Proposition $3 T_{\varepsilon}$ satisfies the principle of progressive transfers if and only if

- $w_{k+1} \leq w_{k}$ and $\varepsilon>1$ or

- $w_{k+1}<w_{k}$ and $\varepsilon=1$

Proof. Consider a progressive transfer from $j$ to $i$ where $x_{i}<x_{j}<x_{n}$. Following Theorem A.4 of Marshall and Olkin (1979) chapter 3, page 57, Axiom 8 requires

$$
\frac{\partial T_{\varepsilon}(\mathbf{x})}{\partial x_{j}}<\frac{\partial T_{\varepsilon}(\mathbf{x})}{\partial x_{i}}
$$


in other words

$$
-T_{\varepsilon}(\mathbf{x})^{1-\varepsilon} w_{j}\left[x_{n}-x_{j}\right]^{\varepsilon-1}<-T_{\varepsilon}(\mathbf{x})^{1-\varepsilon} w_{i}\left[x_{n}-x_{i}\right]^{\varepsilon-1}
$$

or

$$
\frac{w_{i}}{w_{j}}<\left[\frac{x_{n}-x_{j}}{x_{n}-x_{i}}\right]^{\varepsilon-1}
$$

If $\varepsilon>1$ then (14) implies $w_{i} \leq w_{j}$. If $\varepsilon=1$ then (14) implies $w_{i}<w_{j}$. If $\varepsilon<1$ then there is a contradiction.

Proposition 4 Assume that $\mathbb{D}=\Omega_{n}$; then, if $\varepsilon \leq 0, x_{n-1} \rightarrow x_{n}$ implies that $T_{\varepsilon}(\mathbf{x})$ tends to zero.

Proof. $\varepsilon=0$ : obvious.

$\varepsilon<0$ : Note that $T_{\varepsilon}(\mathbf{x})$ is bounded because

$$
0 \leq T_{\varepsilon}(\mathbf{x}) \leq x_{n}-x_{1} .
$$

If $x_{n-1} \rightarrow x_{n}$ then $x_{n}-x_{n-1} \rightarrow 0$; so a limit $A$ of $T_{\varepsilon}(\mathbf{x})$ exists. Suppose $A>0$. Then this would imply

$$
\sum_{k=1}^{n-1} w_{k}\left[x_{n}-x_{k}\right]^{\varepsilon} \rightarrow A^{\varepsilon},
$$

a finite limit. But this implies a contradiction because $\left[x_{n}-x_{n-1}\right]^{\varepsilon} \rightarrow \infty$.

This is a remarkable property which some may find unattractive. However it follows naturally from a value judgment implicit in the choice of $\varepsilon$ - see section 5.2.

\subsection{Extension to $\Omega_{n}^{*}$}

Now consider the general space of income distributions - let $\mathbb{D}=\Omega_{n}^{*}$ in (1). Then we obtain the following analogue to Proposition 1.

Proposition $5\left(\mathbf{1}^{*}\right)$ Assume that $\mathbb{D}=\Omega_{n}^{*}$; then $T$ satisfies Axioms 1 to 6 if and only if there are $w_{k}>0, k=1, \ldots, n-1, \sum_{k=1}^{n-1} w_{k}=1$ and $\varepsilon>0$ such that (8) holds for $\mathbf{x} \in \mathbb{D}$.

In this case we have the convenient property that $T_{\varepsilon}(\mathbf{x})=0$ if and only if all incomes are equal. Analogous counterparts to Propositions 2 and 3 can also easily be written down for the case $\mathbb{D}=\Omega_{n}^{*}$. 


\section{Inequality: discussion}

\subsection{Aggregation of complaints}

We have allowed the aggregation methodology to emerge from the axiomatic structure rather than imposing it directly. A comparison of (8) with the argument in Temkin (1986), pages 111-113 shows that the $T_{\varepsilon}$-class satisfies the weighted additive principle and, for $\varepsilon \neq 1$, the additive principle as a special case.

\subsection{The inequality index}

It is clear that $(8,9)$ represents an absolute inequality index as conventionally understood in the literature. The parameter $\varepsilon$ is an indicator of sensitivity of this index and there are some important special cases contained in the $T_{\varepsilon}$-class $(8,9)$.

- If $\varepsilon \rightarrow \infty$ the inequality measure becomes the range, $x_{n}-x_{1}$; this is of course just the complaint of the poorest person, or max-min inequality (Cf Temkin 1986, page 109).

- Likewise, if $\varepsilon \rightarrow-\infty$ the inequality measure becomes an "upper-middle class" complaint - the complaint of person $n-1$.

- For any $\varepsilon \leq 0$ the sensitivity to inequality takes a very special form, that may be defensible, but is not commonly considered in the mainstream literature - absolute priority is placed on the salience of the topmost person. As we have seen this absolute priority implies that once the gap between the richest and the next richest is closed the distribution is considered to have zero inequality (see the zero-inequality Y-shaped contours in Figure 2 below).

- The case $\varepsilon=1$ yields a generalised (absolute) Gini - see figure 1 . However, there is no admissible system of weights $w_{k}$ that will yield the absolute Gini itself. ${ }^{5}$

${ }^{5}$ As noted by Temkin (1993, page 133) the ATBO notion of complaint will lead to the absolute Gini. See also Yitzhaki (1979). 


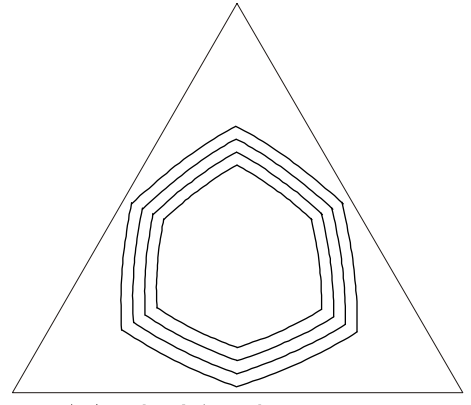

$\mathrm{w} 1=.75$, w $2=.25, \varepsilon=2$

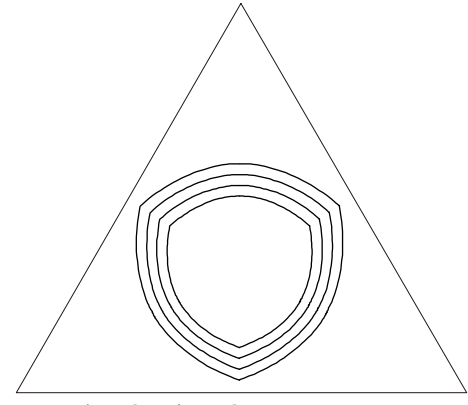

$\mathrm{w} 1=.5, \mathrm{w} 2=.5, \varepsilon=2$

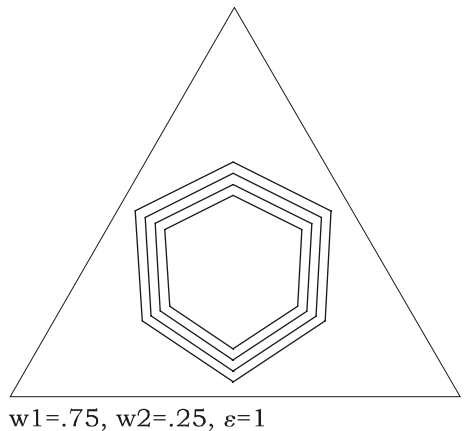

$\mathrm{w} 1=.75, \mathrm{w} 2=.25, \varepsilon=1$

Figure 1: Typical complaint-inequality contours, $\varepsilon>0$.

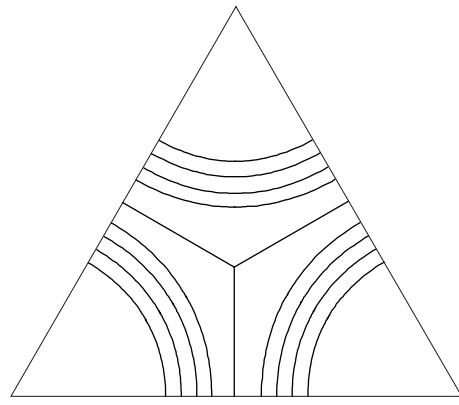

$\mathrm{w} 1=.5, \mathrm{w} 2=.5, \varepsilon=0$

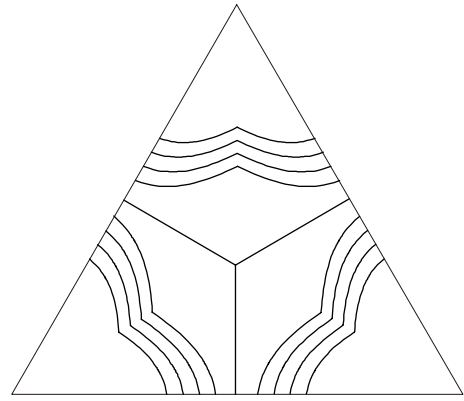

$\mathrm{w} 1=.75$, w2 $=.25, \varepsilon=-1$

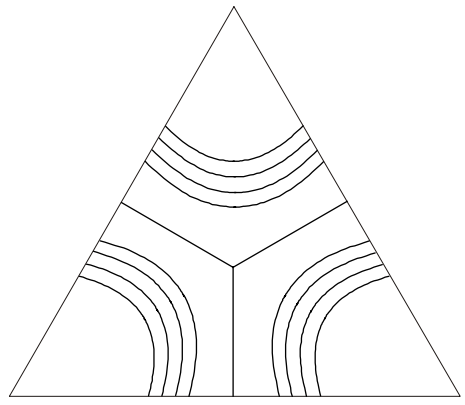

$\mathrm{w} 1=.5, \mathrm{w} 2=.5, \varepsilon=-1$

Figure 2: Typical complaint-inequality contours, $\varepsilon \leq 0$. 
The inequality-contours for four values of $\varepsilon$ and for two cases of the weights are illustrated in Figures 1 and 2. These represent the standard view of the 3-person income distribution map within the simplex for a given value of mean income $\mu$ (see for example Blackorby and Donaldson 1978). Note that all the measures are well-defined for negative incomes; so, although we have restricted the representation of each contour map to the case of positive incomes (the boundary and the interior of the triangle), the contours extend beyond the boundary of the triangle. Given axiom 5 one can generate the corresponding contours for other values of $\mu$ just by resizing the equilateral triangle.

Note that the specification of the inequality measure focuses on the representative income of the reference group rather than the reference group itself - in this case the income $x_{n}$. This is important where there are multiple persons with the income of the richest in order to ensure continuity of the inequality index.

\subsection{The sequence}

Temkin introduces what he calls the "sequence" (Temkin 1986 page 106) in effect a migration story that can be interpreted either as inequality and immiserisation or inequality and income growth. Consider two fixed income levels, $x_{1}$ and $x_{n}\left(>x_{1}\right)$, with $m$ persons on the lower level $x_{1}$ and $n-m$ persons on the upper level $x_{n} ; m$ is variable between 1 and $n-1$ inclusive. What happens to $T_{\varepsilon}$ as $m$ changes?

To borrow Temkin's phrase "world $m$ " can be characterised as an income vector $\mathbf{x}^{m}$ where the components satisfy $x_{n}=\ldots=x_{m+1}>x_{m}=\ldots=x_{1}$. Then for $\varepsilon>0$ (8) yields:

$$
\begin{aligned}
T_{\varepsilon}\left(\mathbf{x}^{m}\right) & =\left[\sum_{k=1}^{m} w_{k}\left[x_{n}-x_{k}\right]^{\varepsilon}\right]^{\frac{1}{\varepsilon}} \\
& =\left[\sum_{k=1}^{m} w_{k}\right]^{\frac{1}{\varepsilon}}\left[x_{n}-x_{1}\right]
\end{aligned}
$$

Given that $w_{k}>0$ it is clear that $T_{\varepsilon}\left(\mathbf{x}^{m}\right)$ is increasing in $m$ if $\varepsilon>0$ (the "worse-and-worse" case of Temkin 1986). For the case $\varepsilon \leq 0$ there is a degenerate sequence in the same direction: inequality is zero for all $m<n-1$ 
and positive for $m=n-1 .^{6}$

However, following Temkin, we have only considered the sequence over unequal worlds - from $m=1$ to $n-1$. What happens at the ends of the sequence corresponding to $m=0$ and $m=n$ ? Clearly inequality is zero in each of these two extremes and because the inequality measure $T_{\varepsilon}$ is monotonic over the sequence it is always true that there will be a discontinuity between $n-1$ and $n$.

\section{Complaint ordering}

Apart from the behaviour of a typical complaint-inequality index it is natural to consider how the concept of complaint may be used more generally in ranking income distributions. To this end introduce:

Definition 4 For any $\mathbf{x} \in D$ define the cumulation of complaints

$$
\left.\begin{array}{l}
d_{0}(\mathbf{x}):=0 \\
d_{i}(\mathbf{x}):=d_{i-1}(\mathbf{x})+x_{n}-x_{i} \quad \text { for } i=1,2, \ldots, n
\end{array}\right\}
$$

This concept can be used to draw the "cumulative complaint contour" (CCC) of a distribution $\mathbf{x}$, formed by joining the points $\left(\frac{i}{n}, d_{i}(\mathbf{x})\right)$; the CCC must be increasing and concave. ${ }^{7}$ Intuitively we can see that if $\operatorname{CCC}(\mathbf{x})$ lies on or above $\mathrm{CCC}(\mathbf{y})$ then distribution $\mathbf{x}$ exhibits more complaint inequality than distribution $\mathbf{y}$. More formally we can characterise an inequality ordering $\succcurlyeq_{T}$ in terms of the complaint cumulations:

Definition $\mathbf{5}$ For any $\mathbf{x}, \mathbf{y} \in D$ distribution $\mathbf{x}$ exhibits more complaintinequality than $\mathbf{y}\left(\mathbf{x} \succcurlyeq_{T} \mathbf{y}\right)$ if and only if

$$
d_{i}(\mathbf{x}) \geq d_{i}(\mathbf{y}) \text { for } i=1,2, \ldots, n
$$

where $d_{i}$ is given by (15).

The ordering $\succcurlyeq_{T}$ is related to familiar concepts in the welfare economics of income distribution, in particular the standard generalised-Lorenz ordering $\succcurlyeq_{\mathrm{GL}}$ (Shorrocks 1983).

\footnotetext{
${ }^{6}$ For further discussion of the "sequence" and inequality see Amiel and Cowell (1994, 1999 pp. 78-86).

${ }^{7}$ This is analogous to the TIP curves of Jenkins and Lambert (1997).
} 
Lemma 6 For any $\mathbf{x}, \mathbf{y} \in D: \mathbf{x} \succcurlyeq_{T} \mathbf{y} \Longleftrightarrow\left[\mathbf{y}-y_{n} \mathbf{1}\right] \succcurlyeq_{\mathrm{GL}}\left[\mathbf{x}-x_{n} \mathbf{1}\right]$.

Proof. By definition 5 we have

$$
\mathbf{x} \succcurlyeq_{T} \mathbf{y} \Longleftrightarrow \sum_{k=1}^{i}\left[x_{n}-x_{k}\right] \geq \sum_{k=1}^{i}\left[y_{n}-y_{k}\right], i=1,2, \ldots, n-1 .
$$

This is equivalent to

$$
\frac{1}{n} \sum_{k=1}^{i}\left[y_{k}-y_{n}\right] \geq \frac{1}{n} \sum_{k=1}^{i}\left[x_{k}-x_{n}\right], i=1,2, \ldots, n
$$

which means that $\left[\mathbf{y}-y_{n} \mathbf{1}\right] \succcurlyeq_{\mathrm{GL}}\left[\mathbf{x}-x_{n} \mathbf{1}\right]$.

Now consider a general class of complaint-inequality indices - those that satisfy the conditions of proposition 3 . Let $\mathcal{T}:=\mathcal{T}_{0} \cup \mathcal{T}_{1}$ where

$$
\begin{gathered}
\mathcal{T}_{0}:=\left\{T_{\varepsilon}: \varepsilon>1, \sum_{k=1}^{n-1} w_{k}=1, w_{k} \geq w_{k+1}>0\right\} \\
\mathcal{T}_{1}:=\left\{T_{1}: \sum_{k=1}^{n-1} w_{k}=1, w_{k}>w_{k+1}>0\right\}
\end{gathered}
$$

There is a close relationship between the class $\mathcal{T}$ and the ordering $\succcurlyeq_{T}$.

Proposition $\mathbf{7}$ For any $\mathbf{x}, \mathbf{y} \in D: \mathbf{x} \succcurlyeq_{T} \mathbf{y} \Longleftrightarrow T_{\varepsilon}(\mathbf{x}) \geq T_{\varepsilon}(\mathbf{y})$, for all $T_{\varepsilon} \in \mathcal{T}$.

Proof. Consider $-T_{\varepsilon}(\cdot)$ as a function of $\mathbf{x}-x_{n} \mathbf{1}$ : it is clearly symmetric, nondecreasing and concave in $\mathbf{x}-x_{n} \mathbf{1}$. So, using Lemma 6 and Theorem 2 of Shorrocks (1983), we find that $\mathbf{x} \succcurlyeq_{T} \mathbf{y}$ implies

$$
-T_{\varepsilon}(\mathbf{y}) \geq-T_{\varepsilon}(\mathbf{x}) \text {. }
$$

Now consider a subfamily of indices with typical member $T^{\alpha, i} \in \mathcal{T}$ where

$$
T^{\alpha, i}(\mathbf{x}):=\sum_{k=1}^{n-1} w_{k}\left[x_{n}-x_{k}\right]
$$




$$
w_{k}=\left\{\begin{array}{ll}
\frac{1}{i}\left[1-\frac{2 \alpha k}{[n-1][i+1]}\right] & \text { for } k=1, \ldots, i \\
\frac{2 \alpha[n-k]}{[n-1][n-i][n-i-1]} & \text { for } k=i+1, \ldots, n-1 .
\end{array}\right\}
$$

Each $T^{\alpha, i}$ is an instance of the case $\varepsilon=1$ in (8). By assumption

$$
T^{\alpha, i}(\mathbf{x}) \geq T^{\alpha, i}(\mathbf{y}), i=1, \ldots, n-1 .
$$

However from (17) and (18) we have

$$
\lim _{\alpha \rightarrow 0} T^{\alpha, i}(\mathbf{x})=d_{i}(\mathbf{x})
$$

and so, letting $\alpha \rightarrow 0$, (19) implies

$$
d_{i}(\mathbf{x}) \geq d_{i}(\mathbf{y}), i=1, \ldots, n-1 .
$$

Hence $\mathbf{x} \succcurlyeq_{T} \mathbf{y}$.

\section{Extensions}

\subsection{Welfare}

As we noted in section 2.3 complaint-inequality was intended to be viewed in terms of "better" or "worse", not just "less" or "more". The analysis in section 4 has focused on the meaning of "more" or "less" inequality in terms of complaints but has not considered the explicit welfare issue. A simple way to incorporate the complaint-inequality index within a standard welfare-economic framework would be to write social welfare as

$$
W(\mathbf{x})=\Phi(X, T)
$$

where

$$
\begin{aligned}
X & =X(\mathbf{x})=\sum_{i=1}^{n} x_{i} \\
T & =T_{\varepsilon}(\mathbf{x})
\end{aligned}
$$



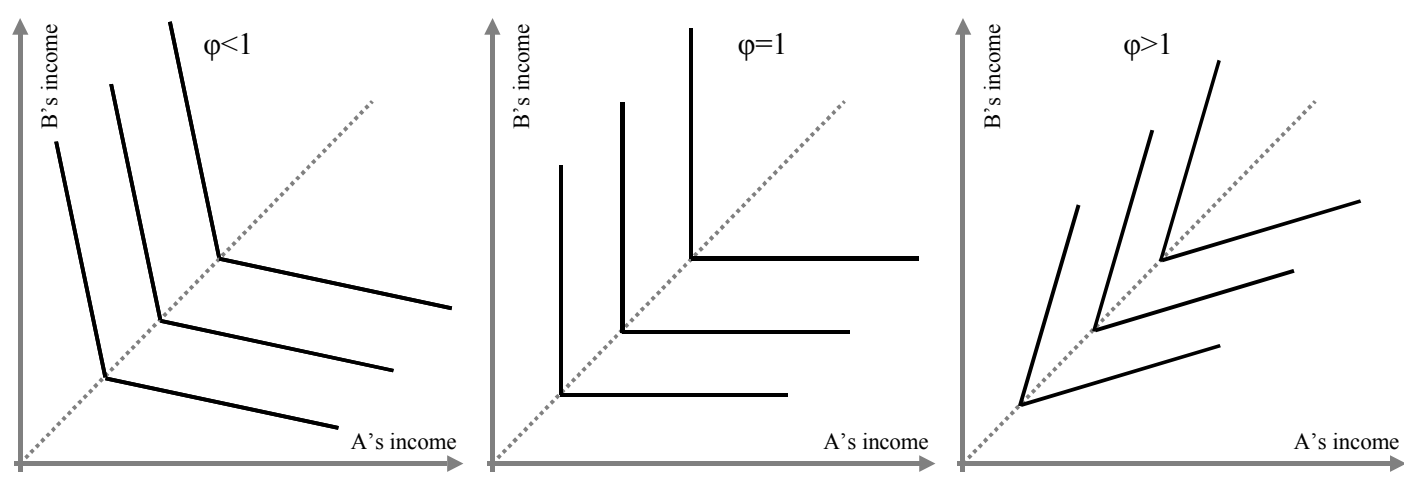

Figure 3: Welfare contours in the case $n=2$

are aggregate income and aggregate complaint, respectively, and $\Phi$ is nondecreasing in its first argument, nonincreasing in its second argument. Some insight can be obtained by taking the linear case of (21):

$$
W=X-\varphi T
$$

where $\varphi>0$ is the marginal value of complaint-inequality, corresponding to $-\frac{\partial \Phi}{\partial T} / \frac{\partial \Phi}{\partial X}$ in (21). In a two-person world we would have

$$
\begin{aligned}
W & =x_{1}+x_{2}-\varphi\left[x_{2}-x_{1}\right] \\
\left.\frac{d x_{2}}{d x_{1}}\right|_{d W=0} & =\frac{1+\varphi}{1-\varphi}
\end{aligned}
$$

for $\varphi \neq 1$. The three principal subcases of this are illustrated in Figure 3. Note that the case $\varphi=1$ yields max-min contours ${ }^{8}$ and that $\varphi>1$ (23) corresponds to Meade's "superegalitarianism" (Meade 1976). The case $n \geq 3$ can be handled similarly.

Temkin (1993), chapter 6 suggests that "inequality matters more in a poor society than in a rich one." This can be interpreted using the welfare framework as the case where $-\frac{\partial \Phi}{\partial T} / \frac{\partial \Phi}{\partial X}$ falls with proportionate increases in $X$ and $T$.

${ }^{8}$ However this only applies for $n=2$. In general BOP is distinct from Rawls' (1971) Maximum Principle - see Temkin (1993), pages 105 ff. 


\subsection{ATBO and AVE}

We have focused on one specific interpretation of the reference group and reference income used to define a complaint. However, the insight from Proposition 1 can be extended to include other concepts.

For a first example define the conditional mean

$$
\mu_{k}(\mathbf{x}):=\frac{1}{n-k} \sum_{h=k+1}^{n} x_{h}
$$

and consider the following family of indices

$$
T_{\varepsilon}^{*}(\mathbf{x})=\left\{\begin{array}{cc}
{\left[\sum_{k=1}^{n-1} w_{k}\left[\mu_{k}(\mathbf{x})-x_{k}\right]^{\varepsilon}\right]^{\frac{1}{\varepsilon}}} & \text { for } \varepsilon \neq 0, \\
\prod_{k=1}^{n-1}\left[\mu_{k}(\mathbf{x})-x_{k}\right]^{w_{k}} & \text { for } \varepsilon=0 .
\end{array}\right.
$$

This is clearly the ATBO counterpart of the family $(8,9)$ and is essentially the same as the deprivation index suggested by Chakravarty and Mukherjee (1999), page 95 .

As a second example take the conventional overall mean $\mu(\mathbf{x})$ and consider the family

$$
T_{\varepsilon}^{* *}(\mathbf{x})=\left\{\begin{array}{cc}
{\left[\sum_{k=1}^{n} w_{k}\left|\mu(\mathbf{x})-x_{k}\right|^{\varepsilon}\right]^{\frac{1}{\varepsilon}}} & \text { for } \varepsilon \neq 0, \\
\prod_{k=1}^{n}\left|\mu(\mathbf{x})-x_{k}\right|^{w_{k}} & \text { for } \varepsilon=0 .
\end{array}\right.
$$

This is an AVE counterpart of the family $(8,9)$, related to the family of "compromise" inequality measures discussed by Ebert (1988). ${ }^{9}$

\subsection{Implementation}

Because the nature of BOP is to focus attention on the richest individual this approach to inequality measurement could attract criticism as to its practicality in empirical application. For some values of $\varepsilon$ there will be a problem of hypersensitivity to small changes in the topmost income and this might

\footnotetext{
${ }^{9}$ However, some caution is necessary here because it is unclear how Temkin intended to treat complaints in the case of AVE where incomes are greater than the mean (Devooght 2001).
} 
mean that inequality estimates could be unreliable when confronted with actual data. There also appears to be a problem of informational requirements in translating the idea of BOP complaints to the real-world: in which communities is it clearly known who the richest person is or what his/her income actually is?

However, these issues are familiar from discussions relating to the implementation of Rawls' (1971) Difference Principle and indeed there are practical work-rounds - see Temkin (1993), pages $111 \mathrm{ff}$. It is clear that the analysis of section 4 would carry over to the case where $n$ individuals were replaced by $\nu$ equal-sized income-ordered groups and inequality within each group were deemed to be irrelevant. For BOP the key concept then becomes the representative income for the topmost group. An appropriate suggestion would be to use the corresponding quantile as the representative income for each group; the case $\varepsilon \rightarrow \infty$ would then be approximated by the difference between extreme quantiles. ${ }^{10}$

\section{Conclusion}

Although Temkin produced some remarkably original insights into the meaning of economic inequality his work left a gap regarding the type of inequality indices that would capture his concept of complaint. We have filled that gap using an axiom system that corresponds to the types of assumption made elsewhere in the literature on the assessment of income distributions. The result is a new class of inequality indices that bears a distinctive relationship to known families of inequality and poverty indices. Associated with that class we also have a new type of ranking principle that neatly embodies the comparison of income distributions in terms of complaints.

\footnotetext{
${ }^{10} \mathrm{Cf}$ the recommendation in Atkinson et al. (2002) pp 126-127. For any $q, 0<q<1$ the $q$ th quantile is an income $x(q):=\min x_{i}: \frac{i}{n} \geq q$.
} 


\section{References}

Aczél, J. (1966). Lectures on Functional Equations and their Applications, Mathematics in Science and Engineering. Number 9. New York: Academic Press.

Amiel, Y. and F. A. Cowell (1994). Inequality changes and income growth. In W. Eichhorn (Ed.), Models and measurement of welfare and inequality, pp. 3-26. Berlin, Heidelberg: Springer-Verlag.

Amiel, Y. and F. A. Cowell (1998). Distributional orderings and the transfer principle: a re-examination. Research on Economic Inequality 8, $195-215$.

Amiel, Y. and F. A. Cowell (1999). Thinking about Inequality. Cambridge: Cambridge University Press.

Atkinson, A. B. (1970). On the measurement of inequality. Journal of Economic Theory 2, 244-263.

Atkinson, A. B., B. Cantillon, E. Marlier, and B. Nolan (2002). Social Indicators: The EU and Social Inclusion. Oxford: Oxford University Press.

Blackorby, C. and D. Donaldson (1978). Measures of relative equality and their meaning in terms of social welfare. Journal of Economic Theory 18, 59-80.

Chakravarty, S. R. (1998). Relative deprivation and satisfaction orderings. Keio Economic Studies 34, 17-31.

Chakravarty, S. R. and D. Mukherjee (1999). Measures of deprivation and their meaning in terms of social satisfaction. Theory and Decision 47 , 89-100.

Cowell, F. A. (2000). Measurement of inequality. In A. B. Atkinson and F. Bourguignon (Eds.), Handbook of Income Distribution, Chapter 2. Amsterdam: North Holland.

Dalton, H. (1920). Measurement of the inequality of incomes. Economic Journal 30(9), 348-361.

Devooght, K. (2001). Measuring inequality by counting 'complaints': theory and empirics. Distributional Analysis Discussion Paper 57, STICERD, London School of Economics, London WC2A 2AE. 
Ebert, U. (1988). A family of aggregative compromise inequality measure. International Economic Review 29(5), 363-376.

Ebert, U. and P. Moyes (2000). An axiomatic characterization of Yitzhaki's index of individual deprivation. Economics Letters 68(3), 263-270.

Ebert, U. and P. Moyes (2002). A simple axiomatization of the FosterGreer-Thorbecke poverty orderings. Journal of Public Economic Theory forthcoming.

Eichhorn, W. (1978). Functional Equations in Economics. Reading Massachusetts: Addison Wesley.

Fishburn, P. C. (1970). Utility theory for decision making. New York: John Wiley.

Foster, J. E., J. Greer, and E. Thorbecke (1984). A class of decomposable poverty measures. Econometrica 52, 761-776.

Jenkins, S. P. and P. J. Lambert (1997). Three 'I's of poverty curves, with an analysis of UK poverty trends. Oxford Economic Papers 49, $317-327$.

Marshall, A. W. and I. Olkin (1979). Inequalities: Theory and Majorization. New York: Academic Press.

Meade, J. E. (1976). The Just Economy, Chapter VII: Measurement and patterns of inequality. Allen and Unwin.

Pigou, A. C. (1912). Wealth and Welfare. London: Macmillan.

Rawls, J. (1971). A Theory of Justice. Cambridge, Massachusetts: Harvard University Press.

Runciman, W. G. (1966). Relative Deprivation and Social Justice. London: Routledge.

Shorrocks, A. F. (1983). Ranking income distributions. Economica 50, 317.

Temkin, L. S. (1986). Inequality. Philosophy and Public Affairs 15, 99-121.

Temkin, L. S. (1993). Inequality. Oxford: Oxford University Press.

Yitzhaki, S. (1979). Relative deprivation and the Gini coefficient. Quarterly Journal of Economics 93, 321-324. 